\title{
Evaluation of chemokine CXCLI 0 in human gingival crevicular fluid, saliva, and serum as periodontitis biomarker
}

This article was published in the following Dove Press journal: Journal of Inflammation Research

\author{
Salwa Aldahlawi' \\ Abdel-Rahman Youssef' \\ Syed Shahabuddin ${ }^{2,3}$ \\ 'Department of Basic and Clinical \\ Oral Sciences, College of Dentistry, \\ Umm Al-Qura University, Makkah, \\ Saudi Arabia; ${ }^{2}$ Abbvie, North Chicago, \\ IL,USA; ${ }^{3}$ Deportment of Biology, City \\ Colleges of Chicago, Chicago, IL, USA
}

Correspondence: Salwa Aldahlawi Department of Basic and Clinical Oral Sciences, College of Dentistry, Umm al-Qura University. PO Box 715, Mecca 21995, Saudi Arabia

$\mathrm{Tel}+966500559365$

Fax +966 I2 5270189

Email sadahlaawi@uqu.edu.sa
Purpose: The aim of this study was to evaluate CXCL10 as a biomarker for periodontitis by determining the CXCL10 levels in saliva, serum, and gingival crevicular fluid (GCF) samples from periodontally healthy control subjects and adult subjects with chronic periodontitis.

Patients and methods: Adult patients seeking dental treatment at Umm Al-Qura University dental clinic underwent a complete periodontal examination, and saliva, serum, and GCF samples were collected. Subjects were classified as chronic periodontitis patients $(n=31)$ if they have a periodontal probing depth (PD) of $\geq 4 \mathrm{~mm}$ and/or clinical attachment level (CAL) of $\geq 3 \mathrm{~mm}$ in $>30 \%$ of the teeth. The control group $(\mathrm{n}=25)$ had $\mathrm{PD} \leq 3 \mathrm{~mm}$ and/or CAL $\leq 2 \mathrm{~mm}$. ELISA was performed to determine the concentration of CXCL10 in saliva, serum, and GCF samples. Student's $t$-test was carried out to evaluate the significant difference between different groups. Spearman's correlation test was used to analyze the relationship between the levels of CXCL10 and the clinical periodontal parameters. $P$-value of $\leq 0.05$ was considered significant.

Results: Significantly higher concentrations of CXCL10 were found in saliva and serum in chronic periodontitis patients as compared with the controls $(272 \pm 60.4 \mathrm{pg} / \mathrm{mL}$ and $72 \pm 13.4 \mathrm{pg} /$ $\mathrm{mL}$ vs $130 \pm 22.2 \mathrm{pg} / \mathrm{mL}$ and $44.08 \pm 4.5 \mathrm{pg} / \mathrm{mL}, P \leq 0.05)$. The CXCL10 levels in GCF were higher in the periodontitis group as compared with the control group $(66.36 \pm 32.0 \mathrm{pg} / \mathrm{mL}$ and $44.56 \pm 17.5$ $\mathrm{pg} / \mathrm{mL}$, respectively); the difference did not reach statistical significance $(P \geq 0.05)$. Moreover, serum CXCL10 level was significantly higher in periodontitis patients with moderate to severe bone loss as compared with those with mild bone loss $(71.05 \pm 4.7 \mathrm{pg} / \mathrm{mL}$ vs $54.8 \pm 7.7 \mathrm{pg} / \mathrm{mL}$, $P \leq 0.05)$. The serum CXCL10 levels were found to be related to CAL measurements $(r=0.3$, $P=0.026)$, while the saliva CXCL10 levels were related to PD measurements ( $r=0.8, P=0.0007)$. Conclusion: CXCL10 is significantly increased in periodontitis subjects as compared with controls and could be used as a marker for periodontal disease.

Keywords: periodontal disease, alveolar bone loss, gingival crevicular fluid, saliva, serum, biomarker, CXCL10, IP-10

\section{Introduction}

Periodontitis is the most common form of inflammatory bone destruction in human beings. It results from the inflammatory response to bacterial challenge in the gingival crevicular area. ${ }^{1}$ Traditional clinical parameters such as probing depth (PD), clinical attachment level (CAL), bleeding on probing (BOP), and radiographic assessment of alveolar bone loss provide information on the severity of the periodontitis. However, these parameters do not measure disease activity, indicate sites for active disease, monitor the response to therapy, or predict susceptibility to future disease progression. The research for a reliable 
biomarker to identify and monitor patients at increased risk of periodontitis as well as to evaluate the disease severity and the response to therapy remains unestablished. ${ }^{2,3}$

Most of the pathology and bone destruction in periodontitis are mediated by an inappropriate immune response. This leads to an imbalance in osteoclastogenesis through the combined action of inflammatory cytokines, chemokines, and $\mathrm{T}$ and B lymphocytes. The circulating cytokines, chemokines, and other mediators have been detected at elevated levels in the gingival crevicular fluid (GCF) and saliva of patients who have periodontal disease making them putative biomarkers of the disease. ${ }^{4-9}$ Although each mediator holds the promise to be a potential biomarker, no definite marker has been established.

Chemokine CXCL10, also known as IP-10, appears to have a direct influence on osteoclastogenesis for its involvement in leukocyte and osteoclast precursor diapedesis ${ }^{10}$ and subsequent presence in the periodontal environment. CXCL10 is a $10 \mathrm{kDa}$ protein and functionally categorized as an "inflammatory" chemokine. It is secreted by various cell types, such as monocytes, neutrophils, endothelial cells, keratinocytes, fibroblasts, mesenchymal cells, dendritic cells, and astrocytes, and it binds to CXCR3. It regulates immune responses by activating and recruiting leukocytes, such as T cells, eosinophils, monocytes, and natural killer cells. ${ }^{11-13}$ Recent reports have shown that the serum and tissue expression of CXCL10 are increased in periodontitis. ${ }^{14-17}$ CXCL10 has also been found to stimulate osteoclastogenesis and bone resorption largely through upregulation of RANKL in rheumatoid arthritis (RA).${ }^{18-20}$ Furthermore, augmentation of CXCL10 and RANKL appears to promote osteoclast differentiation and osteolytic bone metastasis. ${ }^{21}$ These data indicate that CXCL10 plays an important role in leukocyte homing to inflamed tissues and in the perpetuation of inflammation and thus may significantly contribute to tissue damage via RANKL, which is responsible for periodontal bone resorption. ${ }^{22-24}$

CXCL10 has shown promise as a potential biomarker for the detection of periodontitis and post-therapy monitoring; however, no definite correlation has been established between CXCL10 levels in serum, saliva, and GCF with disease severity. ${ }^{14,16}$

The aim of this study was to evaluate CXCL10 as a biomarker for periodontitis by determining the CXCL10 levels in GCF, saliva, and serum samples from chronic periodontitis and control adult subjects.

\section{Patients and methods}

The study protocol was approved by the ethics committee for clinical research at Umm Al-Qura University Faculty of
Dentistry (UQUDENT). The study population was derived from subjects seeking treatment at the UQUDENT clinic, and all participants provided a written informed consent prior to the enrollment in the study. All subjects were healthy and did not receive periodontal treatment during the past 3 months. Subjects who had any systemic disease, reported the use of anticonvulsant or immunosuppressive drugs, received antibiotic treatment or periodontal treatment in the past 3 months, or were pregnant were excluded from the study.

All subjects underwent a complete periodontal examination performed by a calibrated examiner. Full mouth plaque index (PI), gingival index (GI), and clinical periodontal measurements (PD and CAL) were obtained from 4 points of each tooth: mesial, distal, lingual (palatal), and labial (buccal) excluding third molars. The PD and CAL were evaluated by a manual periodontal probe. The PD was defined as the distance between the gingival margins to the base of the sulcus. The CAL was determined from the cementoenamel junction (CEJ) to the base of the sulcus; if the CEJ cannot be determined, then a fixed point was used for CAL measurement. The GI was used to evaluate the degree of inflammation, with a value of 0 given to normal gingiva, 1 for mild inflammation but no BOP, 2 for moderate inflammation and BOP, and 3 for gingiva exhibiting severe inflammation with a tendency to bleed spontaneously. The PI values are described as follows: 0 , no plaque; 1 , when a film of plaque is adhering to free gingival margin and adjacent area and can be seen only by disclosing tablet; 2, moderate accumulation of deposits which can be seen by the naked eye; and 3, abundant soft material along the gingival margin.

Orthopantomographic (OPG) radiographs were reviewed from the patients' records to determine the radiographic bone level. Smoking was evaluated based on the personal report of tobacco smoking in the past 2 years.

According to the periodontal and radiographic examination, subjects were classified into the following groups:

- Chronic periodontitis group: At the time of the oral examination, the chronic periodontitis group had a CAL of $\geq 3$ $\mathrm{mm}$ and/or $\mathrm{PD}$ of $\geq 4 \mathrm{~mm}$ in $>30 \%$ of the teeth. In addition, they demonstrated horizontal or vertical alveolar bone loss in the OPG (the radiographic distance between the bone crest and CEJ junction was $\geq 3 \mathrm{~mm}$ at more than $30 \%$ of the sites).

- Control group: At the time of the oral examination, the subjects in this group had healthy gingiva or varying degrees of gingival inflammation, $\mathrm{CAL}$ of $\leq 2 \mathrm{~mm}$ or PD of $\leq 3 \mathrm{~mm}$, and no indication of horizontal or vertical alveolar bone loss in the radiographic examinations (the 
radiographic distance between the bone crest and CEJ was $<3 \mathrm{~mm}$ at $90 \%$ of the sites).

\section{Saliva collection}

All subjects were instructed not to eat or use any oral hygiene measures for at least 2 hours before collection. They had to rinse their mouths with water at the beginning of the appointment, and saliva was collected after 10 minutes by the passive drooling method. About $3 \mathrm{~mL}$ of the un-stimulated whole - mixed - saliva sample was collected in $50 \mathrm{~mL}$ sterile collection tubes and placed on ice. The supernatant was collected by centrifugation at 3,800 rpm for 10 minutes and stored at $-80^{\circ} \mathrm{C}$ until analysis.

\section{Site selection and GCF sampling}

GCF samples were collected from four Ramfjord teeth in the control group and from four teeth showing the deepest periodontal PD in the chronic periodontitis group. Each tooth zone was dried by air spraying to prevent irritation and isolated carefully with cotton tampons. To avoid contamination of samples with saliva, saliva absorbents were used. Standard paper strips (Periopaper; Oraflow, Plainview, NY, USA) were placed in the mesial or distal parts of the sulcus by pushing until resistance was felt. Each paper strip was kept in the sulcus for 30 seconds, and the GCF samples were compared with a reference guide. The volume of GCF on each strip was determined following the technique reported by Alencar et $\mathrm{al}^{25}$ and then immediately transferred to the Eppendorf tubes and placed on ice. Samples were kept at $-80^{\circ} \mathrm{C}$ until analysis. Four samples, one from each of the four teeth of every subject, were collected. All four samples were pooled and eluted in $250 \mu \mathrm{L}$ of PBS. The CXCL10 concentration in each patient's GCF was expressed in picogram/milliliter after multiplying with the dilution factor.

\section{Blood sampling}

Five milliliters of venous blood sample was collected in plain vacutainer tubes using a standard venipuncture method and was immediately transferred to the laboratory. The blood samples were allowed to clot at room temperature and after 1 hour; the serum was separated by centrifugation at 4,000 rpm for 10 minutes. The serum was transferred to storage vials and stored in sterile Eppendorf tubes at $-80^{\circ} \mathrm{C}$ until the day of laboratory analysis.

\section{ELISA analysis of CXCLIO}

The levels of CXCL10 were analyzed in GCF, saliva, and serum using a commercial ELISA kit (Abcam, Cambridge,
UK). All samples were thawed at the room temperature. GCF was eluted from the Periopaper by adding $250 \mu \mathrm{L}$ of PBS and mixing on Vortex. Standards in the commercial ELISA kit were diluted, and test samples were added to wells coated with CXCL10-specific antibodies. All assay procedures were performed according to the manufacturer's instructions, and the absorbance values were determined by a spectrophotometric ELISA Reader (SpectroStar; BMG LABTECH, Offenburg, Germany). The total amounts of CXCL10 were expressed as picogram $/$ milliliter.

The data were analyzed using the SPSS statistical package software, for Windows version 17.0 (SPSS Inc., Chicago, IL, USA). All the quantitative data are presented as mean and SD. Student's $t$-test was carried out to evaluate the significant difference between the different groups. Spearman's correlation test was used to analyze the relationship between the levels of CXCL10 and clinical periodontal parameters. $P$-value of $\leq 0.05$ was considered significant.

\section{Results}

A total of 56 subjects participated in the study with a mean age of 30 years (range 18-59 years). There was female predominance among the participants with 40 female and 16 male subjects. A total of six subjects were identified as current smokers with an average 10 pack-years of smoking (Table 1).

Thirty-one subjects were classified as chronic periodontitis patients, and 25 subjects were classified as the control group. The average PD in the chronic periodontitis group was $4.4 \pm 1.0 \mathrm{~mm}$ and CAL was $3.1 \pm 1.42 \mathrm{~mm}$. The PI was 2 , and GI was 2.4. In the control group, the average PD was $3.2 \pm 0.4 \mathrm{~mm}$ and the CAL was $0.36 \pm 0.4 \mathrm{~mm}$. The PI was 1.9 , and GI was 2.5. The difference between the two groups was highly significant with regard to $\mathrm{PD}$ and CAL $(P=0.0001$ and $P=0.001$, respectively; Table 2 ). The chronic periodontitis group had an average of $18 \%$ of sites with deep PD of $5 \mathrm{~mm}$ or more (range $3-55 \%$ ), while the percentage of CAL $\geq 3 \mathrm{~mm}$ was $30 \%$ (range $3-100 \%$ ). This was significantly different from the control group ( $P=0.0001$; Table 2$)$.

Table I Demographic characteristics of control and chronic periodontitis subjects

\begin{tabular}{|c|c|c|c|}
\hline & & $\begin{array}{l}\text { Chronic } \\
\text { periodontitis }\end{array}$ & Control \\
\hline Mean ag & & 31.7 & 27.8 \\
\hline Range & & $18-59$ & $18-48$ \\
\hline Gondor & Female & 20 & 20 \\
\hline Gender & Male & 11 & 5 \\
\hline Smoking & & 6 & 0 \\
\hline
\end{tabular}


Table 2 Clinical periodontal parameters in chronic periodontitis and control subjects

\begin{tabular}{|l|l|l|}
\hline & $\begin{array}{l}\text { Chronic } \\
\text { periodontitis }\end{array}$ & Control \\
\hline PD (mean \pm SD) & $4.4 \pm 1.0$ & $3.0 \pm 0.4^{\mathrm{a}}$ \\
\hline Clinical attachment loss (mean \pm SD) & $3.1 \pm 1.4$ & $0.36 \pm 0.4^{\mathrm{b}}$ \\
\hline Percentage of PD $\geq 5 \mathrm{~mm}$ & $18 \%(3-55)$ & $1 \%$ \\
\hline Percentage of CAL $\geq 3 \mathrm{~mm}$ & $30 \%(3-100)$ & $0.8 \%^{\mathrm{d}}$ \\
\hline $\mathrm{PI}$ & 2 & 1.9 \\
\hline $\mathrm{Gl}$ & 2.4 & 2.5 \\
\hline
\end{tabular}

Notes: a Mean PD in the periodontitis vs control groups: $P=0.0001$. 'Mean attachment level in the periodontitis vs control groups: $P=0.00$ I. 'Percentage of sites with $P D \geq 5 \mathrm{~mm}$ in the periodontitis vs control groups: $P=0.00 \mathrm{I}$. ${ }^{\mathrm{d}}$ Percentage of sites with $C A L \geq 3$ in the periodontitis vs control groups: $P=0.000$ I.

Abbreviations: CAL, clinical attachment level; GI, gingival index; PD, probing depth; PI, plaque index.

Variation in the CXCL10 levels in saliva, serum, and GCF is shown in Figure 1. In the chronic periodontitis group, the mean CXCL10 saliva concentration was $272 \pm 308 \mathrm{pg} / \mathrm{mL}$. The corresponding value for controls was $130 \pm 49 \mathrm{pg} / \mathrm{mL}$, significantly lower as compared with the periodontitis group $(P=0.017)$. The CXCL10 concentration in serum was $72 \pm 66$ $\mathrm{pg} / \mathrm{mL}$ and $44.08 \pm 18 \mathrm{pg} / \mathrm{mL}$ in the chronic periodontitis and the control groups, respectively, and the difference was statistically significant $(P=0.028)$. Although the CXCL10 level in GCF was higher in the periodontitis group as compared with the control group $(66.36 \pm 132 \mathrm{pg} / \mathrm{mL}$ and $44.56 \pm 70$ $\mathrm{pg} / \mathrm{mL}$, respectively), the difference did not reach statistical significance $(P=0.27)$.

The number of smokers in the study population was low, and they had similar clinical parameters compared with nonsmokers ( $4.0 \pm 0.2 \mathrm{~mm}$ for PD and $2.7 \pm 0.2 \mathrm{~mm}$ for CAL). However, the average CXCL10 concentration in saliva was greater in smokers compared with nonsmokers (374 \pm 150 $\mathrm{pg} / \mathrm{mL}$ and $240.5 \pm 61.6 \mathrm{pg} / \mathrm{mL}$, respectively; Figure 2). The same was noted for CXCL10 concentration in serum and GCF $(99.2 \pm 55.0 \mathrm{pg} / \mathrm{mL}$ vs $67.6 \pm 4.1 \mathrm{pg} / \mathrm{mL}$ and $97.0 \pm 79.2$ $\mathrm{pg} / \mathrm{mL}$ vs $63.3 \pm 40.0 \mathrm{pg} / \mathrm{mL}$ ). The statistical difference in the CXCL10 levels between the smokers and nonsmokers could not be generated due to the small smoking population size.

The chronic periodontitis group was divided according to the severity of bone loss as estimated on OPG radiographs into the mild bone loss group $(\mathrm{n}=20)$ and the moderate to severe bone loss group $(\mathrm{n}=10)$. The average $\mathrm{PD}$ was deeper in the moderate to severe group as compared with the mild group ( $4.86 \pm 1.44 \mathrm{~mm}$ vs $4.02 \pm 0.56 \mathrm{~mm}, P=0.05$ ). Similarly, CAL was worse in the moderate to severe group $(4.1 \pm 1.2 \mathrm{~mm}$ vs $2.2 \pm 1.2 \mathrm{~mm}$ in the mild group, $P=0.0002$ ). The serum CXCL10 levels were significantly higher in the moderate to severe bone loss group $(71.05 \pm 4.7 \mathrm{pg} / \mathrm{mL}$ vs $54.8 \pm 7.7 \mathrm{pg} /$ $\mathrm{mL}, P=0.02)$. Although the CXCL10 levels in saliva and GCF were higher in the moderate to severe bone loss group (300.9 $\pm 116.9 \mathrm{pg} / \mathrm{mL}$ and $116 \pm 99.3 \mathrm{pg} / \mathrm{mL}$, respectively) as compared with the mild periodontitis group $(233.8 \pm 69.8 \mathrm{pg} /$ $\mathrm{mL}$ and $51.1 \pm 30.8 \mathrm{pg} / \mathrm{mL}$, respectively), the difference did not reach statistical significance $(P=0.3$ and $P=0.4$, respectively; Figure 3).

When the chronic periodontitis group was divided by age (younger $<30$ years old, $n=13$, and older $>30$ years old, $\mathrm{n}=17$ ), the average PD was found to be similar in both groups ( $4.0 \pm 0.3 \mathrm{~mm}$ and $4.0 \pm 0.2 \mathrm{~mm}$ for younger and older subjects). However, the older subjects had a significantly higher CAL when compared with the younger subjects $(3.2 \pm 0.3 \mathrm{~mm}$ vs $2.0 \pm 0.4 \mathrm{~mm}, P=0.01)$. Serum CXCL10 was significantly higher in the older group $(67.9 \pm 7.4 \mathrm{pg} / \mathrm{mL}$ vs $50.6 \pm 7.3 \mathrm{pg} / \mathrm{mL}$, $P=0.05)$. Although the CXCL10 concentrations in saliva and GCF did not reach the level of significance, all were higher in the older group compared with the younger group (249 \pm 106 $\mathrm{pg} / \mathrm{mL}$ vs $284.9 \pm 81.3 \mathrm{pg} / \mathrm{mL}, P=0.39$ for saliva $)$ and $(61.7 \pm 44.1$ $\mathrm{pg} / \mathrm{mL}$ vs $76.6 \pm 67.4 \mathrm{pg} / \mathrm{mL}, P=0.41$ for GCF; Figure 4).

Spearman's rank correlation was performed to analyze the associations between the CXCL10 levels in serum, saliva, and GCF and clinical parameters (PD, CAL). The serum CXCL10 levels were found to be related to CAL measurements $(r=0.3, P \leq 0.05)$, while the saliva CXCL10 levels were related to PD measurements $(r=0.8, P \leq 0.001)$. However, the levels of CXCL10 in GCF were not related to any of the clinical measurements.

\section{Discussion}

CXCL10 has been shown to play a role in progenitor osteoclast recruitment and activation of RANKL expression which, upon binding to its receptor RANK on the progenitor osteoclast, converts them into a mature osteoclast and leads to bone resorption in periodontitis. Thus, CXCL10 may be used as a biomarker for periodontitis. The current study was designed to compare the mean levels of CXCL10 (IP10) in GCF, saliva, and serum samples from periodontally healthy and chronic periodontitis subjects and examine the correlations between CXCL10 and clinical parameters of periodontal disease.

The results demonstrated that the chronic periodontitis subjects had significantly higher levels of the CXCL10 in saliva and serum than the periodontally healthy subjects. There were significant positive associations among the clinical parameters of periodontal disease and CXCL10. Serum CXCL10 level was significantly higher in the moderate to 
A

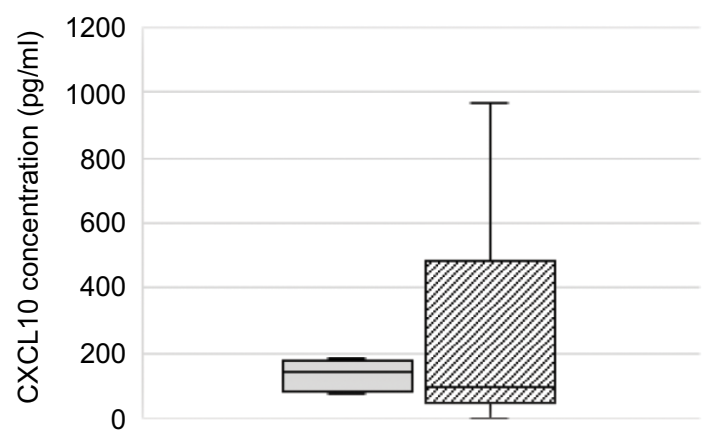

$\square$ Control

Chronic periodontitis

B

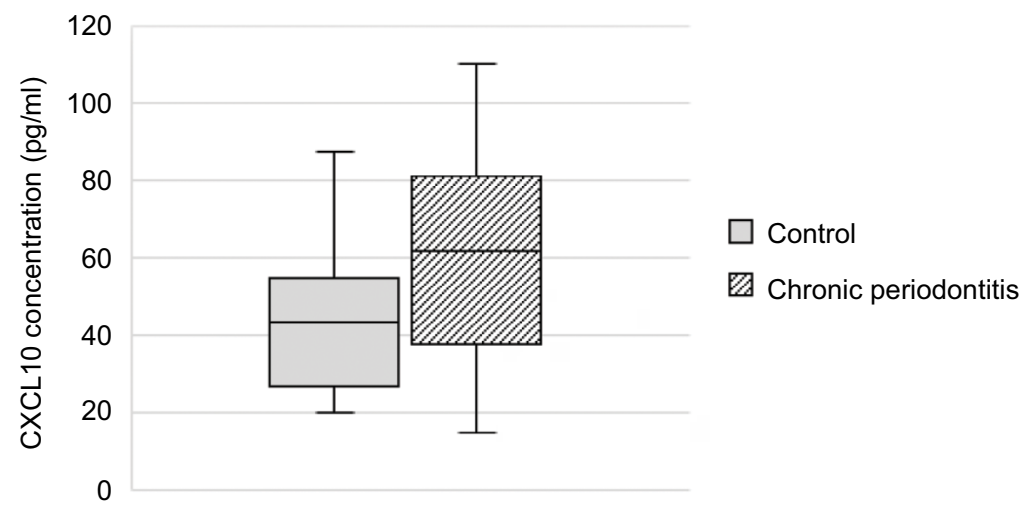

C

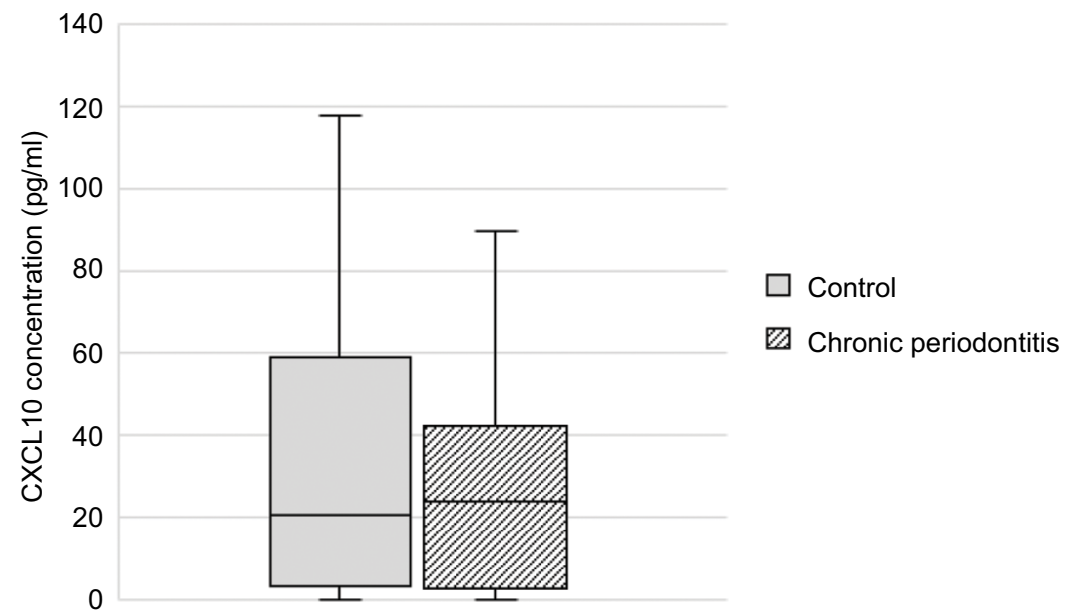

Figure I CXCLIO concentrations ( $\mathrm{pg} / \mathrm{mL})$ in (A) saliva, (B) serum, and (C) GCF form the chronic periodontitis and control groups. The horizontal line represents the median value, and $75 \%$ and $25 \%$ quartiles are represented by the upper and lower edges of boxes, respectively. CXCLI 0 levels in saliva and serum in the chronic periodontitis vs control groups were different at statistically significant level $(P<0.05)$.

Abbreviation: GCF, gingival crevicular fluid. 


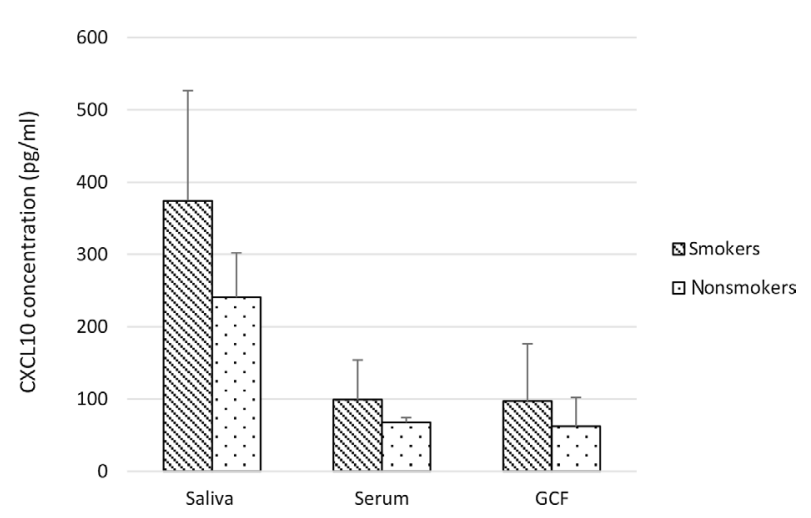

Figure 2 CXCLI 0 levels in saliva, serum, and GCF among smoker and nonsmoker subjects with chronic periodontitis.

Abbreviation: GCF, gingival crevicular fluid.

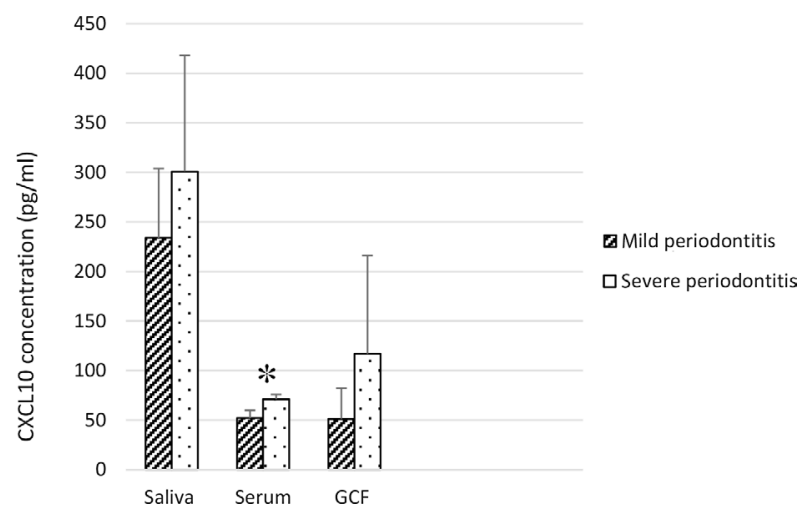

Figure 3 CXCLIO levels in saliva, serum, and GCF among subjects with mild periodontitis and moderate to severe periodontitis. $* P \leq 0.05$.

Abbreviation: GCF, gingival crevicular fluid.

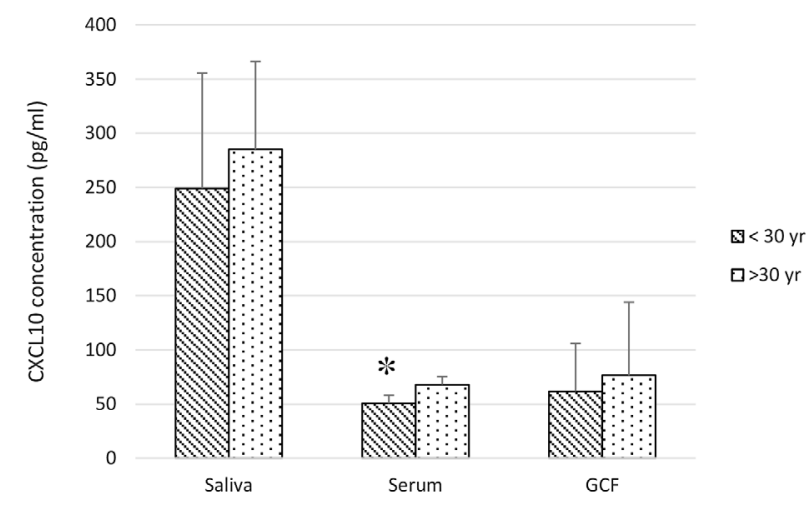

Figure $4 \mathrm{CXCLIO}$ levels in saliva, serum, and GCF among chronic periodontitis subjects of the two age groups. ${ }_{P \leq 0.05}$.

Abbreviations: GCF, gingival crevicular fluid; yr, year.

severe periodontitis group as defined by deeper PD and worse CAL as compared with the mild periodontitis group. Furthermore, the serum CXCL10 was higher in older subjects who had a significantly higher attachment loss when compared with younger subjects.
Although GCF level of CXC10 was higher in the periodontitis group and in subjects with moderate to severe periodontitis as compared with the mild periodontitis group, the difference did not reach statistical significance.

These findings reinforce previous reports of associations between the CXCL10 levels and clinical signs of periodontal disease. ${ }^{14-17}$ In addition, this is the first ever finding that directly shows the difference in the CXCL10 and severity of bone loss in periodontitis. ${ }^{18,20}$ Furthermore, this is the first report on the significant positive associations among clinical parameters of periodontal disease and CXCL10 among the Saudi population. ${ }^{19}$

The relevance of CXCL10 in periodontitis vis-a-vis bone loss has been demonstrated in several studies. Recent studies in mice observed an increase in CXCL10 in high bone loss strain but not in the low bone loss strain after LPS injections. Genetic deletion of CXCR3 (CXCL10 receptor) demonstrated about $50 \%$ reduction in bone loss and reduced osteoclasts after LPS injections. Furthermore, wild-type mice treated with a CXCR3 antagonist showed about 45\% reduction in bone loss and decreased osteoclasts after LPS injections. These studies suggested CXCR3 as a strong candidate for modulating the host response in individuals susceptible to periodontitis. ${ }^{26}$

In another study with a gene-knockout mouse model, CXCL10 was demonstrated to be associated with RANKLmediated osteoclastogenesis. ${ }^{27}$ Serum CXCL10 levels were elevated in those animals with increased osteoclastic activities. Furthermore, CXCL10 genes were highly expressed in those mice that showed increased osteoclastic activities in in vitro quantification of bone resorption. The increased serum level of CXCL10 in chronic periodontitis patients in the current study is supported by others, and its importance is underscored in osteoclastogenesis. In an in vitro study, peripheral mononuclear cells (PBMCs) from patients with chronic periodontitis produced higher levels of CXCL10 than the controls. ${ }^{28}$ In addition, osteoclast differentiation was induced by RANKL alone in PBMCs from patients with chronic periodontitis, but not in PBMCs from the healthy controls. Furthermore, PBMC-derived osteoclasts from patients with chronic periodontitis showed significantly higher resorption activity than those observed in the healthy controls. Taken together, this directly supports our finding of significantly higher serum CXCL10 levels in chronic periodontal subjects compared with non-periodontal subjects and its relevance in osteoclastogenesis.

Support for our findings also comes from earlier findings where CXCL10 was evaluated as a biomarker in periodontitis 
by Shimada et $\mathrm{al}^{14}$ who found GCF IP-10 (CXCL10) levels to be significantly higher in BOP-positive diseased sites than BOP-negative diseased sites, after adjustment for multiple comparisons. More recently, a correlation of serum chemokines with periodontal disease parameters was published. It showed elevated serum CXCL10 levels in periodontal disease which support our data. ${ }^{16}$

The role of CXCL10 in bone resorption is well documented in other diseases particularly in RA. ${ }^{18-20}$ Lee et al have reported that CXCL10 increased RANKL expression in CD4+ T cells, and it was mediated by G (i) subunits of CXCR3. They suggested that CXCL10 may have a potential role in the osteoclastogenesis of RA synovial tissue and subsequent joint erosion. ${ }^{29}$ With regard to CXCL10's role in osteoclast recruitment, a recent report shows that chemokine signals are crucial for enhanced homing and differentiation of circulating osteoclast progenitor cells. ${ }^{10}$ The study identified distinct effects of selected chemokines on the stimulation of osteoclast progenitor cell mobilization, tissue homing, and maturation.

Studies on bone metastasis show that CXCL10 facilitates the trafficking of CXCR3-expressing cancer cells to bone, which augments its own production and promotes osteoclastic differentiation via RANKL-mediated osteoclast formation. Therefore, CXC10 may represent a therapeutic target for osteolytic bone metastasis. ${ }^{21}$

To the best of our knowledge, this is the first report showing increased CXCL10 levels in chronic periodontitis compared with control subjects, correlating the systemic levels and local levels of CXCL10 with the severity of the periodontal disease. As for the differences in smokers and nonsmokers with regard to CXCL10 levels, there appears to be a trend of variable activity among the smokers as shown by others, ${ }^{30}$ but more subjects need to be tested before a statistical conclusion can be derived. Likewise, more subjects need to be studied to establish an age-related difference in the CXCL10 level, even though serum CXCL10 level was significantly higher in the older age group ( $>30$ years old) compared with the younger age group ( $<30$ years old). The results support the fact that the older subjects may have more advanced periodontal disease as indicated by higher CAL; after all, periodontal disease may be worse at older ages as a cumulative effect of long-term damage.

Clinical diagnosis of periodontal disease relies on the clinical measurement of periodontal pocket depth and clinical attachment loss. Reliability of those measurements in detecting or predicting future disease progression has been addressed extensively in the periodontal literature. ${ }^{31}$ In general, current diagnostic methods reflect the past disease experience rather than ongoing disease activities. The use of oral fluids may provide diagnostic and prognostic markers for periodontal disease. However, with multifactorial diseases such as periodontal disease, it is unlikely that one single molecule will be reliable to characterize periodontal disease. On the contrary, a complex network of biomarkers is involved and needs to be investigated to allow for patientspecific clinical management. ${ }^{32}$

\section{Conclusion}

Our findings show higher serum, saliva, and GCF CXCL10 levels in chronic periodontitis subjects than control subjects and a direct correlation of increased CXCL10 levels with the disease severity. These findings may correlate CXCL10 with alveolar bone loss occurring during periodontal disease through its interaction with RANKL and regulation of RANK/RANKL/OPG axis in osteoclastogenesis. Based on these findings, CXCL10 can be used as one of the biomarkers for periodontitis.

\section{Acknowledgment}

This study was supported by the Institute of Scientific Research (grant no.: 43409058) from Umm Al-Qura University, Makkah, Saudi Arabia.

\section{Author contributions}

SA contributed to conception and design, acquisition, analysis and interpretation of data, drafting the article, and revising it. ARY contributed to acquisition, analysis and interpretation of data, drafting the article, and revising it critically for important intellectual content. SS contributed to conception and design, acquisition, analysis and interpretation of data, drafting the article, and revising it critically for important intellectual content. All the authors are accountable for all aspects of the work and read and approved this version of the manuscript.

\section{Disclosure}

SS was a project manager contractor at Abbvie. The authors report no other conflicts of interest in this work.

\section{References}

1. Seymour GJ. Possible mechanisms involved in the immunoregulation of chronic inflammatory periodontal disease. J Dent Res. 1987;66(1): 2-9.

2. Barros SP, Williams R, Offenbacher S, Morelli T. Gingival crevicular fluid as a source of biomarkers for periodontitis. Periodontol 2000. 2016;70(1):53-64. 
3. Korte DL, Kinney J. Personalized medicine: an update of salivary biomarkers for periodontal diseases. Periodontol 2000. 2016;70(1):26-37.

4. Stadler AF, Angst PD, Arce RM, Gomes SC, Oppermann RV, Susin C. Gingival crevicular fluid levels of cytokines/chemokines in chronic periodontitis: a meta-analysis. J Clin Periodontol. 2016;43(9):727-745.

5. Lamster IB. Evaluation of components of gingival crevicular fluid as diagnostic tests. Ann Periodontol. 1997;2(1):123-137.

6. Sexton WM, Lin Y, Kryscio RJ, Dawson DR 3rd, Ebersole JL, Miller CS. Salivary biomarkers of periodontal disease in response to treatment. J Clin Periodontol. 2011;38(5):434-441.

7. Gemmell E, Carter CL, Seymour GJ. Chemokines in human periodontal disease tissues. Clin Exp Immunol. 2001;125(1):134-141.

8. Miller CS, King CP Jr, Langub MC, Kryscio RJ, Thomas MV. Salivary biomarkers of existing periodontal disease: a cross-sectional study. $J$ Am Dent Assoc. 2006;137(3):322-329.

9. Gonçalves L da R, Soares MR, Nogueira FC, et al. Comparative proteomic analysis of whole saliva from chronic periodontitis patients. $J$ Proteomics. 2010;73(7):1334-1341.

10. Sucur A, Jajic Z, Artukovic M, et al. Chemokine signals are crucial for enhanced homing and differentiation of circulating osteoclast progenitor cells. Arthritis Res Ther. 2017;19(1):142.

11. Yu X, Graves DT. Fibroblasts, mononuclear phagocytes, and endothelial cells express monocyte chemoattractant protein-1 (MCP-1) in inflamed human gingiva. J Periodontol. 1995;66(1):80-88.

12. Luster AD, Ravetch JV. Biochemical characterization of a gamma interferon-inducible cytokine (IP-10). J Exp Med. 1987;166(4):1084-1097.

13. Taub DD, Lloyd AR, Conlon K, et al. Recombinant human interferoninducible protein 10 is a chemoattractant for human monocytes and $\mathrm{T}$ lymphocytes and promotes T cell adhesion to endothelial cells. $J$ Exp Med. 1993;177(6):1809-1814.

14. Shimada Y, Tabeta K, Sugita N, Yoshie H. Profiling biomarkers in gingival crevicular fluid using multiplex bead immunoassay. Arch Oral Biol. 2013;58(6):724-730.

15. Jinquan T, Jing C, Jacobi HH, et al. CXCR3 expression and activation of eosinophils: role of IFN-gamma-inducible protein-10 and monokine induced by IFN-gamma. J Immunol. 2000;165(3):1548-1556.

16. Panezai J, Ghaffar A, Altamash M, Sundqvist KG, Engström PE, Larsson A. Correlation of serum cytokines, chemokines, growth factors and enzymes with periodontal disease parameters. PLoS One. 2017;12(11): e0188945.

17. Vernal R, Chaparro A, Graumann R, Puente J, Valenzuela MA, Gamonal J. Levels of cytokine receptor activator of nuclear factor kappaB ligand in gingival crevicular fluid in untreated chronic periodontitis patients. J Periodontol. 2004;75(12):1586-1591.
18. Kwak HB, Ha H, Kim HN, et al. Reciprocal cross-talk between RANKL and interferon-gamma-inducible protein 10 is responsible for boneerosive experimental arthritis. Arthritis Rheum. 2008;58(5):1332-1342.

19. Lee JH, Kim B, Jin WJ, Kim HH, Ha H, Lee ZH. Pathogenic roles of CXCL10 signaling through CXCR3 and TLR4 in macrophages and T cells: relevance for arthritis. Arthritis Res Ther. 2017;19(1):163.

20. Hanaoka R, Kasama T, Muramatsu M, et al. A novel mechanism for the regulation of IFN-gamma inducible protein-10 expression in rheumatoid arthritis. Arthritis Res Ther. 2003;5(2):R74-81.

21. Lee JH, Kim HN, Kim KO, et al. CXCL10 promotes osteolytic bone metastasis by enhancing cancer outgrowth and osteoclastogenesis. Cancer Res. 2012;72(13):3175-3186.

22. Jin Q, Cirelli JA, Park CH, et al. RANKL inhibition through osteoprotegerin blocks bone loss in experimental periodontitis. J Periodontol. 2007;78(7):1300-1308.

23. Almehmadi AH, Alghamdi F. Biomarkers of alveolar bone resorption in gingival crevicular fluid: A systematic review. Arch Oral Biol. 2018;93:12-21.

24. Han X, Kawai T, Eastcott JW, Taubman MA. Bacterial-responsive B lymphocytes induce periodontal bone resorption. J Immunol. 2006;176(1):625-631.

25. Alencar FG, Kronbauer GC, Gomes SC. Validation of an alternative device for volumetric quantification of crevicular fluid. Acta Odontol Latinoam. 2011;24(1):29-34.

26. Hiyari S, Green E, Pan C, et al. Genomewide Association Study Identifies Cxcl Family Members as Partial Mediators of LPS-Induced Periodontitis. J Bone Miner Res. 2018;33(8):1450-1463.

27. Yim HY, Park C, Lee YD, et al. Elevated Response to Type I IFN Enhances RANKL-Mediated Osteoclastogenesis in Usp18-Knockout Mice. J Immunol. 2016;196(9):3887-3895.

28. Herrera BS, Bastos AS, Coimbra LS, et al. Peripheral blood mononuclear phagocytes from patients with chronic periodontitis are primed for osteoclast formation. J Periodontol. 2014;85(4):e72-e81.

29. Lee EY, Seo M, Juhnn YS, et al. Potential role and mechanism of IFNgamma inducible protein-10 on receptor activator of nuclear factor kappa-B ligand (RANKL) expression in rheumatoid arthritis. Arthritis Res Ther. 2011;13(3):R104.

30. Tymkiw KD, Thunell DH, Johnson GK, et al. Influence of smoking on gingival crevicular fluid cytokines in severe chronic periodontitis. $J$ Clin Periodontol. 2011;38(3):219-228.

31. Beltrán-Aguilar ED, Eke PI, Thornton-Evans G, Petersen PE. Recording and surveillance systems for periodontal diseases. Periodontol 2000. 2012;60(1):40-53.

32. Bostanci N, Belibasakis GN. Gingival crevicular fluid and its immune mediators in the proteomic era. Periodontol 2000. 2018;76(1):68-84.
Journal of Inflammation Research

\section{Publish your work in this journal}

The Journal of Inflammation Research is an international, peer-reviewed open access journal that welcomes laboratory and clinical findings on the molecular basis, cell biology and pharmacology of inflammation including original research, reviews, symposium reports, hypothesis formation and commentaries on: acute/chronic inflammation; mediators of

\section{Dovepress}

inflammation; cellular processes; molecular mechanisms; pharmacology and novel anti-inflammatory drugs; clinical conditions involving inflammation. The manuscript management system is completely online and includes a very quick and fair peer-review system. Visit http://www.dove press.com/testimonials.php to read real quotes from published authors. 